\title{
Determinantes sociales que condicionan la actividad sexual precoz de los adolescentes
}

\section{Social determinants that condition early sexual activity of adolescents}

\author{
Sonia Benita Fernández Tapia « 1,a
}

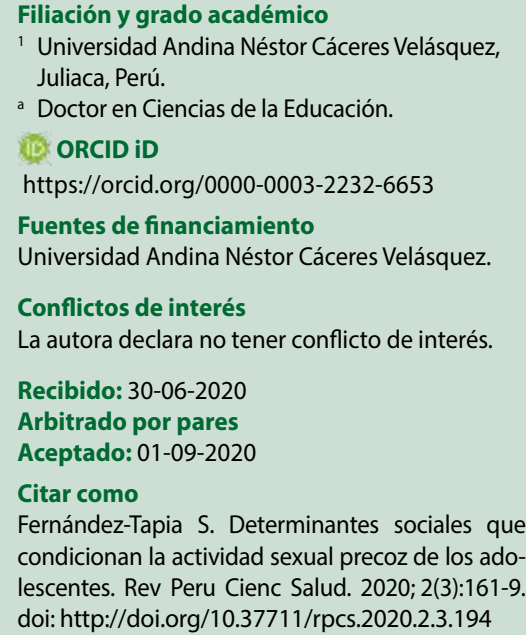

\section{RESUMEN}

Objetivo. Analizar los determinantes sociales que condicionan la actividad sexual precoz de los adolescentes. Métodos. Estudio cuantitativo, descriptivo, explicativo y transversal. La muestra estuvo constituida por 254 estudiantes de 14 a 18 años de edad, a quienes se aplicó un cuestionario que evaluó las dimensiones personales, familiares, contextuales y el inicio de la actividad sexual. Para el análisis de la relación entre variables se utilizó la prueba estadística ji-cuadrado y modelo de regresión lineal múltiple. Resultados. Se identificaron los condicionantes personales, la edad de inicio sexual, la falta de uso de anticonceptivos y el poco conocimiento de los signos y síntomas de las infecciones de transmisión sexual. Entre los determinantes familiares, el grado de instrucción de los padres, el apoyo de la mamá y la violencia verbal y física aparecen como los que tienen una asociación significativa. Junto a la falta de hábito en acudir a los servicios de salud, los programas de televisión y los amigos aparecen como los que más promueven el inicio de la actividad sexual precoz. Conclusión. Los determinantes personales, familiares y contextuales influyen significativamente en el inicio de la actividad sexual de los adolescentes, atentando en la salud sexual y reproductiva de estos.

Palabras clave: humanos, adolescente, salud reproductiva, estudios transversales, amigos, madres, abuso físico, modelos lineales, determinantes sociales de la salud, salud sexual, enfermedades de transmisión sexual, comportamiento de anticoncepción, estudiantes, encuestas y cuestionarios, hábitos, estado educativo, agentes anticonceptivos (Fuente: DeCS - BIREME).

\section{ABSTRACT}

Objective. To analyze the social determinants that condition early sexual activity of adolescents. Methods. Quantitative, descriptive, explanatory and cross-sectional study. The sample comprised 254 students between 14 and 18 years of age. A questionnaire was applied to them. It evaluated the personal, family and contextual dimensions and the initiation of sexual activity. For the analysis of the relationship between variables, chi-square statistical test and multiple linear regression model were used. Results. Personal conditioning factors, age of sexual initiation, lack of contraceptive use, and little knowledge of the signs and symptoms of sexually transmitted infections were identified. Among determinants related to their families, level of education of their parents, support from their mothers, and verbal and physical violence appear to have a significant association. Along with the lack of habit of going to health services, television programs and friends appear as the ones that most promote the initiation of early sexual activity. Conclusions. Personal, family and contextual are determinants that significantly influence initiation of adolescent sexual activity, affecting their sexual and reproductive health.

Keywords: Humans, Adolescent, Reproductive Health, Cross-Sectional Studies, Friends, Mothers, Physical Abuse, Linear Models, Social Determinants of Health, Sexual Health, Sexually Transmitted Diseases, Contraception Behavior, Students, Surveys and Questionnaires, Habits, Educational Status, Contraceptive Agents (Source: MeSH - NLM). 


\section{INTRODUCCIÓN}

La adolescencia es una etapa de la vida comprendida entre los 10 y 19 años, que merece especial atención, ya que se trata de un periodo donde no se ha alcanzado la madurez física, cognoscitiva ni emocional, capaz de responsabilizarse de sus actos y sus consecuencias ${ }^{(1)}$; de modo que, al tener relaciones sexuales sin haber recibido información adecuada de salud reproductiva, la inadecuada comunicación con sus padres, la violencia y las infecciones de transmisión sexual (ITS), terminan resultando un riesgo para su salud ${ }^{(2,3)}$.

Estudios realizados en América Latina reflejan la prevalencia de actividad sexual, siendo la edad promedio de inicio en 15 años para el 52,8 \% (4). En "Chile los adolescentes inician su actividad sexual a una edad promedio entre 15,7 y 16,3 años para los varones y entre 15,5 y 17,1 años para las mujeres" (5,6). En el Perú los resultados oscilan entre los 14 y 16 años ${ }^{(7,8)}$. La gran mayoría de los estudiantes no busca información relacionada con la sexualidad o piensan que lo saben todo y no son conscientes de los riesgos, viviendo el presente sin preocuparse del mañana; de tal modo que usan el condón, "a veces sí y a veces no" (9). Esta situación conduce a embarazos en la adolescencia; un riesgo en la salud, tanto para la joven madre como para el/la recién nacido/a, incluyendo los riesgos del aborto; así como la aparición de múltiples obstáculos para continuar la formación escolar o para el acceso al trabajo.

La sexualidad es parte integral de la personalidad y está presente en todo el ciclo del desarrollo de la vida de la persona desde su nacimiento ${ }^{(10)}$. Los determinantes personales con la edad como indicador de mayor fuerza que explica el inicio de la actividad sexual, varían de una cultura a otra y se encuentran relacionados con factores ambientales y socio culturales ${ }^{(11-13)}$. El uso de los métodos anticonceptivos es fundamental en la salud reproductiva; de modo que, al no tener una adecuada información, los adolescentes actuarán según sus criterios y no utilizarán los anticonceptivos en forma correcta, lo que acabará repercutiendo en un embarazo no deseado, o contagio ITS $/ \mathrm{VIH}^{(14,15)}$, afectando a su salud sexual y reproductiva ${ }^{(16)}$. El consumo de bebidas alcohólicas es una conducta que, por diversos motivos, realizan para sentirse alegres, seguros, en familiaridad, facilitando la interacción grupal, disminuyendo la angustia psicológica, agregándose a las particularidades propias de la edad del adolescente como la de conocer y experimentar nuevas sensaciones; lo que favorece el inicio de la vida sexual del adolescente bajo los efectos del alcohol (17).

El determinante familiar y la educación de los padres es elemental en el comportamiento sexual; así que, por medio de una comunicación positiva se debe promover el dialogo manteniendo la confianza, afecto y comunicación abierta, en temas de sexualidad que ellos deseen que se les aclaren, con una información veraz y no distorsionada ${ }^{(18,19)}$.

La exposición constante a la violencia familiar a una edad muy temprana tiene alcances negativos en el desarrollo cognitivo, afectivo y la salud. Sus consecuencias se manifiestan más adelante en la violencia de pareja, siendo este un fenómeno social problemático en el que los adolescentes pueden verse involucrados ${ }^{(20)}$. Existen determinantes contextuales como la presión de los amigos o enamorados, con quienes comparten más su tiempo y tienen confianza hacia ellos, por lo que toman en cuenta su opinión ${ }^{(21)}$. Del mismo modo, los medios de comunicación son también instancias que favorecen el acercamiento a temas sexuales que generan en el estudiante motivación en la actividad sexual.

Otro determinante es la posibilidad de llegar a los servicios de salud que es de particular trascendencia. En ese sentido, la Organización Mundial de la Salud exhorta: garantizar el acceso a servicios de salud apropiados y orientados al adolescente" y tengan las consideraciones básicas para otorgar un servicio "amigable" por lo tanto "brindar una atención a sus necesidades específicas, que sean equitativos, accesibles, aceptables, y eficaces ${ }^{(22)}$.

El Ministerio de Educación no cuenta actualmente con datos a los que este estudio se orienta, serán de gran beneficio y reorientación de las políticas públicas para los adolescentes presentes y futuros. El propósito de la investigación es analizar los determinantes sociales que condicionan la actividad sexual precoz en los adolescentes.

\section{MÉTODOS}

\section{Diseño del estudio}

Investigación de tipo cuantitativo, descriptivo, con corte explicativo y transversal, realizada en las instituciones educativas secundarias públicas de la ciudad de Puno.

\section{Población y muestra}

En el estudio participaron todas/os las/os adolescentes del quinto año de secundaria de cinco instituciones educativas secundarias de la ciudad de Puno, con edades comprendidas entre los 14 y los 18 años. Con una población de 745 estudiantes, se utilizó la técnica de muestreo aleatorio simple con un nivel de confianza del $95 \%$ y un error de estimación del $5 \%$ de la cual se obtuvo una muestra de 254 adolescentes. 


\section{Criterios de selección}

Los criterios de Inclusión fueron: todos los adolescentes comprendidos entre las edades de 10 y 19 años que posean la información de las variables de interés. Los criterios de exclusión, por su parte, fueron: aquellos estudiantes que están en la etapa joven y adulta.

\section{Variables de medición}

La técnica empleada fue la encuesta y, el instrumento, un cuestionario diseñado y autoadministrado. Para medir la fiabilidad del instrumento se evaluó la consistencia interna (coeficiente alfa de Cronbach 0.742), con una prueba piloto de 50 estudiantes con características similares a la población de estudio. Las dimensiones incluidas en el instrumento se dividen en determinantes personales, familiares y contextuales.

Variables personales: se consideran el sexo y la edad; se evaluó con la escala tipo Likert cuyas opciones de reacción o respuesta en temas de infecciones de transmisión sexual de la mujer y del varón fue "nada" $=0$, "poco" $=1$, "moderado" $=2$, "bastante" $=3$ y "mucho" $=4$. El consumo de alcohol fue "nunca le han ofrecido" $=1$, "al menos una vez" $=2$, "siempre" $=3$, "algunas veces" $=4 \mathrm{y}$ "una vez" $=5$.

Para la variable familia se considera el grado de instrucción de la madre y el padre, el estado afectivo y la comunicación con padres; se evaluó con la escala Likert: "muy bien" = 5, "bien" = 4, "regular" = 3, "mal" = 2 y "muy mal" $=1$. La violencia verbal y física es por el amigo/a, enamorado/a, papá, mamá.

Para analizar la variable dimensión contextual se considera lo referente al acceso a servicios de salud:"no acude" $=1$, "algunas veces" $=2$, "siempre" $=3$. Al medir a la variable amigos (Likert) se le pregunta: ¿cuando hablas con tu amigo/as sobre sexualidad, se resuelven tus dudas? "definitivamente si" $=1$, "probablemente $\mathrm{si}^{\prime \prime}=2$, "indeciso" = 3 , "probablemente no" $=4$, "definitivamente no" $=5$. ¿Al hablar con tus amigos/as sobre relaciones sexuales sientes curiosidad por experimentar? Las alternativas son: "definitivamente si" $=1$, "probablemente si" $=2$, "indeciso" $=3$, "probablemente no" $=4$, "definitivamente no" $=5$. ¿Consideras que los programas que ves en la televisión tienen contenido sexual?:"definitivamente si" = 1 , "probablemente $\mathrm{si}$ " $=2$, "indeciso" $=3$, "probablemente no" $=4$, "definitivamente no" $=5$.

Para la variable "actividad sexual" se recogió la edad de inicio de la actividad sexual precoz.

\section{Proceso de recolección de datos}

Con la información obtenida se creó la base de datos, los cuales fueron ordenados en tablas de frecuencias $y$ estadísticos descriptivos básicos. Posteriormente, para el análisis bivariado se utilizó la prueba de ji-cuadrado y el modelo de regresión lineal múltiple. Los resultados se analizaron a través del programa SPSS versión 23.

\section{Aspectos éticos}

Se tomaron en cuenta los principios éticos de la declaración de Helsinki. La aplicación del instrumento fue a los adolescentes con el diligenciamiento de un consentimiento informado y no se solicitaron datos personales o de filiación de los adolescentes que lo hicieron de manera voluntaria.

\section{RESULTADOS}

En la tabla 1 se contó para la participación de 254 adolescentes en edades de 14 a 18 años $(M=16,12$; $\mathrm{DE}=0,64)$. Como se muestra en la tabla 1 , la muestra estuvo conformada en un $62,6 \%$ por el género femenino y en $37,4 \%$ por el masculino. Respecto a la edad de los adolescentes la mayoría de encuestados tienen 16 años (65\%). La edad de inicio de la actividad sexual se tuvo en la adolescencia temprana en un 9,1\% (47) de casos, y en la adolescencia en etapa tardía en un 9,5\% observándose que un $81,5 \%$ de adolescentes no iniciaron aún su actividad sexual.

Los resultados de la tabla 2, al analizar con el chicuadrado, nos muestran que existe una gran significación de la variable edad del adolescente y el inicio de la actividad sexual (chi-square $=508,000, p=0,00$ ), existe también una gran asociación con la edad (chisquare $=35,219, p=0,00$ ). La variable de uso de métodos

Tabla 1. Frecuencias de las variables individuales de adolescentes de las IES, Puno, 2019

\begin{tabular}{lcc}
\hline Variable & $\mathbf{f i}$ & $\%$ \\
\hline Género & 159 & 62,6 \\
Femenino & 95 & 37,4 \\
Masculino & & \\
Edad de los adolescentes & 1 & 0,4 \\
14 & 30 & 11,8 \\
15 & 165 & 64,9 \\
16 & 53 & 20,9 \\
17 & 5 & 1,9 \\
18 & & \\
Actividad sexual & 207 & 81,5 \\
No tuvo actividad sexual & 23 & 9,05 \\
Adolescencia temprana (10-14 años) & 24 & 9,5 \\
Adolescencia tardía (15-19 años) &
\end{tabular}


Tabla 2. Determinantes personales que condicionan la actividad sexual de adolescentes de las IES, Puno, 2019

\begin{tabular}{|c|c|c|}
\hline \multicolumn{2}{|r|}{ Actividad sexual } & \multirow[b]{2}{*}{ Valor-p } \\
\hline $\begin{array}{l}\text { No tuvo } \\
\text { actividad } \\
\text { sexual }\end{array}$ & $\begin{array}{c}\text { Adolescencia Adolescencia } \\
\text { temprana tardía }\end{array}$ & \\
\hline
\end{tabular}

Dimensión personal

Edad de inicio de actividad sexual

$\begin{array}{lllll}0 & 81,5 \% & 0,0 \% & 0,0 \% & \\ 13 & 0,0 \% & 1,6 \% & 0,0 \% & \mathrm{Chi}^{2}=508,000 \\ 14 & 0,0 \% & 7,5 \% & 0,0 \% & \mathrm{p}=, 000 \\ 15 & 0,0 \% & 0,0 \% & 7,1 \% & \\ 16 & 0,0 \% & 0,0 \% & 2,0 \% & \\ 17 & 0,0 \% & 0,0 \% & 0,4 \% & \end{array}$

Edad de los adolescentes

$\begin{array}{lllll}14 & 0,4 \% & 0,0 \% & 0,0 \% & \\ 15 & 8,3 \% & 0,8 \% & 2,8 \% & \\ 16 & 55,9 \% & 4,7 \% & 4,3 \% & \mathrm{Chi}^{2}=35,219 \\ 17 & 16,1 \% & 2,4 \% & 2,0 \% & \mathrm{p}=, 000 \\ 18 & 0,8 \% & 1,2 \% & 0,4 \% & \end{array}$

Uso de métodos anticonceptivos

$\begin{array}{llllc}\text { No responde } & 79,9 \% & 5,1 \% & 2,4 \% & \\ \text { Píldora } & 0,0 \% & 0,4 \% & 0,8 \% & \\ \text { Inyectable } & 0,0 \% & 0,4 \% & 0,0 \% & \text { Chi }^{2}=143,202 \\ \text { Preservativo } & 0,0 \% & 2,0 \% & 4,7 \% & \mathrm{p}=, 000 \\ \text { Retiro } & 0,0 \% & 0,0 \% & 0,4 \% & \\ \text { No usa } & 1,6 \% & 1,2 \% & 1,2 \% & \end{array}$

Reconocimiento de infección de transmisión en la mujer

$\begin{array}{lcccc}\text { Nada } & 2,0 \% & 1,2 \% & 1,6 \% & \\ \text { Poco } & 41,3 \% & 2,8 \% & 0,8 \% & \mathrm{Chi}^{2}=27,901 \\ \text { Moderado } & 26,8 \% & 4,3 \% & 4,7 \% & \mathrm{p}=, 000 \\ \text { Bastante } & 11,4 \% & 0,8 \% & 2,4 \% & \end{array}$

Reconocer cuando un hombre tiene una infección transmisión sexual o venérea

\begin{tabular}{|c|c|c|c|c|}
\hline Nada & $3,1 \%$ & $1,6 \%$ & $2,0 \%$ & \multirow{4}{*}{$\begin{array}{c}\mathrm{Chi}^{2}=19,614 \\
p=, 01\end{array}$} \\
\hline Poco & $49,6 \%$ & $3,9 \%$ & $1,2 \%$ & \\
\hline Moderado & $22,0 \%$ & $3,1 \%$ & $4,3 \%$ & \\
\hline Bastante & $6,7 \%$ & $0,4 \%$ & $2,0 \%$ & \\
\hline \multicolumn{5}{|c|}{ Ofrecimiento de alcohol } \\
\hline $\begin{array}{l}\text { Nunca le } \\
\text { han ofrecido }\end{array}$ & $43,7 \%$ & $3,1 \%$ & $1,2 \%$ & \multirow{4}{*}{$\begin{aligned} C h i^{2} & =19,581 \\
p & =, 001\end{aligned}$} \\
\hline $\begin{array}{l}\text { Al menos } \\
\text { una vez }\end{array}$ & $22,0 \%$ & $4,3 \%$ & $4,7 \%$ & \\
\hline $\begin{array}{l}\text { Siempre o } \\
\text { casi siempre }\end{array}$ & $4,3 \%$ & $0,0 \%$ & $1,2 \%$ & \\
\hline $\begin{array}{l}\text { Algunas } \\
\text { veces }\end{array}$ & $11,4 \%$ & $1,6 \%$ & $2,4 \%$ & \\
\hline
\end{tabular}

anticonceptivos (chi-square $=143,202, p=0,00$ ), indica una fuerte significancia. Existe una importante significancia en reconocimiento de infección de transmisión sexual en la mujer (chi-square $=27,901, p=0,000$ ), al contrario de reconocer cuando un hombre tiene una infección de transmisión sexual (chi-square $=19,6014, p=0,01$ ), los cuales poco saben de ITS. En cuanto a la variable ofrecimiento de alcohol (chi-square $=19,581, p=0,001$ ), tiene gran significancia en los grupos que nunca le ofrecieron.

En la tabla 3, los datos estadísticos descriptivos de las variables "familia", el nivel más alto en la media se centra en el apoyo de la mamá, con un 3,61 y el más bajo en el grado de estudios de la mamá, con una media de 1,84.

En la tabla 4 se observa la variable "familia" y su asociación con la actividad sexual. La mayoría de los padres tienen estudios secundarios y existe una mayor significancia en el grado de estudios de papá (chisquare $=19,200, p=0,001)$, frente al grado de estudios de mamá (chi-square $=13,150, p=0,01$ ). Además, tiene una asociación significancia el apoyo de mamá (chisquare $=19,614, p=0,01$ ). El apoyo de su padre (chisquare $=25,379, p=0,001$ ) tiene una significancia fuerte. La agresión verbal (chi-square $=45,581, p=0,000$ ) tiene una fuerte significación, al igual que la agresión física (chi-square $=27,283, p=0,001$ ).

En la tabla 5 se observa la variable contextual que condiciona la actividad sexual. Al respecto, se observa si los amigos resuelven las dudas sobre la sexualidad (chisquare $=15,673, p=0,047$ ) evidenciándose una fuerte relación, pero aún más la curiosidad por experimentar (chi-square $=64,950, p=0,000$ ). Los medios de comunicación donde se promueven más las relaciones sexuales (chi-square $=26,701, p=0,001$ ) tienen fuerte significación, al igual que los programas de televisión (chi-square $=39,295, p=0,000$ ), mientras que en los servicios de salud para adolescentes (chi-square $=10,069$, $p=0,01$ ) la significancia es menor.

En cuanto a las variables predictoras de la actividad sexual mostradas en la tabla 6, en este análisis de regresión

Tabla 3. Estadísticos Descriptivos de las variables Familia de los adolescentes de las IES, Puno, 2019

\begin{tabular}{lcc}
\hline Variable & Media & Desviación estándar \\
\hline Grado de estudios mamá & 1,84 & 0,79 \\
Grado de estudios papá & 2,36 & 0,93 \\
Apoyo mamá & 3,61 & 1,02 \\
Apoyo papá & 3,04 & 1,19 \\
\hline
\end{tabular}


Tabla 4. Determinantes familiares que condicionan la actividad sexual de adolescentes de las IES, Puno, 2019

\begin{tabular}{|c|c|c|c|c|c|}
\hline & \multicolumn{4}{|c|}{ Actividad sexual } & \multirow[b]{2}{*}{ Valor-p } \\
\hline & $\begin{array}{c}\text { No tuvo actividad } \\
\text { sexual }\end{array}$ & \multicolumn{2}{|c|}{ Adolescencia temprana } & Adolescencia tardía & \\
\hline \multicolumn{6}{|c|}{ Grado de estudios de papá } \\
\hline Primaria & $11,4 \%$ & \multicolumn{2}{|c|}{$3,1 \%$} & $1,2 \%$ & $\begin{aligned} \mathrm{Chi}^{2} & =19,200 \\
\mathrm{p} & =, 001\end{aligned}$ \\
\hline Secundaria & $43,3 \%$ & \multicolumn{2}{|c|}{$1,6 \%$} & $3,1 \%$ & \\
\hline Instituto & $15,7 \%$ & \multicolumn{2}{|c|}{$2,8 \%$} & $2,0 \%$ & \\
\hline Universidad & $11,0 \%$ & \multicolumn{2}{|c|}{$1,6 \%$} & $3,1 \%$ & \\
\hline \multicolumn{6}{|c|}{ Grado de estudios de mamá } \\
\hline Primaria & $27,6 \%$ & \multicolumn{2}{|c|}{$4,7 \%$} & $4,7 \%$ & $\mathrm{Chi}^{2}=13,150$ \\
\hline Secundaria & $40,9 \%$ & \multicolumn{2}{|c|}{$2,8 \%$} & $2,0 \%$ & $p=, 01$ \\
\hline Instituto & $10,6 \%$ & \multicolumn{2}{|c|}{$0,8 \%$} & $2,4 \%$ & \\
\hline Universidad & $2,4 \%$ & \multicolumn{2}{|c|}{$0,8 \%$} & $0,4 \%$ & \\
\hline \multicolumn{6}{|c|}{ Apoyo de mamá o persona que hace de ella } \\
\hline Muy mal & $2,0 \%$ & \multicolumn{2}{|c|}{$0,0 \%$} & $0,4 \%$ & $\mathrm{Chi}^{2}=19,614$ \\
\hline Mal & $4,3 \%$ & \multicolumn{2}{|c|}{$0,4 \%$} & $0,8 \%$ & $p=, 01$ \\
\hline Regular & $34,3 \%$ & \multicolumn{2}{|c|}{$7,1 \%$} & $6,7 \%$ & \\
\hline Bien & $15,7 \%$ & \multicolumn{2}{|c|}{$0,8 \%$} & $0,4 \%$ & \\
\hline Muy bien & $25,2 \%$ & \multicolumn{2}{|c|}{$0,8 \%$} & $1,2 \%$ & \\
\hline \multicolumn{6}{|c|}{ Apoyo de papá o persona que hace de él } \\
\hline Muy mal & $10,2 \%$ & \multicolumn{2}{|c|}{$1,2 \%$} & $1,6 \%$ & $\mathrm{Chi}^{2}=25,379$ \\
\hline Mal & $13,0 \%$ & \multicolumn{2}{|c|}{$0,8 \%$} & $2,0 \%$ & $p=, 001$ \\
\hline Regular & $27,6 \%$ & \multicolumn{2}{|c|}{$6,3 \%$} & $5,9 \%$ & \\
\hline Bien & $16,9 \%$ & \multicolumn{2}{|c|}{$0,4 \%$} & $0,0 \%$ & \\
\hline Muy bien & $13,8 \%$ & \multicolumn{2}{|c|}{$0,4 \%$} & $0,0 \%$ & \\
\hline \multicolumn{6}{|l|}{ Agresión verbal } \\
\hline Amigo(a) & $11,4 \%$ & $0,4 \%$ & $0,0 \%$ & & \\
\hline Enamorado(a) & $6,7 \%$ & $0,8 \%$ & $0,4 \%$ & $C h i^{2}=45,581$ & \\
\hline Papá & $20,1 \%$ & $5,9 \%$ & $7,9 \%$ & $\mathrm{p}=, 000$ & \\
\hline Mamá & $12,6 \%$ & $0,8 \%$ & $0,4 \%$ & & \\
\hline Ninguna & $30,7 \%$ & $1,2 \%$ & $0,8 \%$ & & \\
\hline \multicolumn{6}{|l|}{ Agresión física } \\
\hline Amigo(a) & $15,7 \%$ & $0,4 \%$ & $0,0 \%$ & & \\
\hline Enamorado(a) & $20,1 \%$ & $0,8 \%$ & $0,4 \%$ & $C h i^{2}=27,283$ & \\
\hline Papá & $25,6 \%$ & $4,7 \%$ & $7,1 \%$ & $p=, 001$ & \\
\hline Mamá & $10,2 \%$ & $2,0 \%$ & $1,2 \%$ & & \\
\hline Ninguna & $9,8 \%$ & $1,2 \%$ & $0,8 \%$ & & \\
\hline
\end{tabular}

múltiple por procedimientos, las variables que mejor predicen el nivel de actividad sexual son: el apoyo de mamá (a mayor apoyo menor nivel de actividad sexual) con un p-valor de 0,001 y los medios de comunicación que promueven más las relaciones sexuales (a mayores medios de comunicaciones que promuevan las relaciones sexuales mayor actividad sexual) con un p-valor de 0,017. Sin embargo, la variable que mayor predice es el apoyo de mamá.

\section{DISCUSIÓN}

Los determinantes sociales impactan sobre la salud sexual y reproductiva de este grupo etario en el inicio de su actividad sexual. Los resultados de este estudio confirman que un determinante personal como la edad es una variable significativa que explica el inicio de la actividad sexual. Los adolescentes encuestados refieren haber iniciado su actividad sexual desde los 13 a los 
Tabla 5. Determinantes contextuales que condicionan la actividad sexual de adolescentes de la IES, Puno, 2019

\begin{tabular}{|c|c|c|c|c|}
\hline & \multicolumn{3}{|c|}{ Actividad sexual } & \multirow[b]{2}{*}{ Valor-p } \\
\hline & $\begin{array}{c}\text { No tuvo actividad } \\
\text { sexual }\end{array}$ & $\begin{array}{l}\text { Adolescencia } \\
\text { temprana }\end{array}$ & $\begin{array}{l}\text { Adolescencia } \\
\text { tardía }\end{array}$ & \\
\hline \multicolumn{5}{|c|}{ Amigos/as sobre sexualidad se resuelven las dudas } \\
\hline Definitivamente sí & $14,6 \%$ & $0,8 \%$ & $2,0 \%$ & \multirow{5}{*}{$\begin{aligned} \mathrm{Chi}^{2} & =15,673 \\
p & =, 047\end{aligned}$} \\
\hline Probablemente sí & $20,1 \%$ & $3,9 \%$ & $2,0 \%$ & \\
\hline Indeciso & $19,7 \%$ & $2,0 \%$ & $0,0 \%$ & \\
\hline Probablemente no & $16,1 \%$ & $0,8 \%$ & $3,1 \%$ & \\
\hline Definitivamente no & $11,0 \%$ & $1,6 \%$ & $2,4 \%$ & \\
\hline \multicolumn{5}{|c|}{ Amigos/as sobre relaciones sexuales y la curiosidad por experimentar } \\
\hline Definitivamente sí & $8,3 \%$ & $4,3 \%$ & $6,3 \%$ & \multirow{5}{*}{$\begin{aligned} \mathrm{Chi}^{2} & =64,950 \\
\mathrm{p} & =, 000\end{aligned}$} \\
\hline Probablemente sí & $8,3 \%$ & $0,8 \%$ & $0,8 \%$ & \\
\hline Indeciso & $17,3 \%$ & $2,0 \%$ & $2,0 \%$ & \\
\hline Probablemente no & $17,3 \%$ & $1,2 \%$ & $0,0 \%$ & \\
\hline Definitivamente no & $30,3 \%$ & $0,8 \%$ & $0,4 \%$ & \\
\hline \multicolumn{5}{|c|}{ Medio de comunicación que promueve más las relaciones sexuales } \\
\hline Internet & $62,2 \%$ & $8,7 \%$ & $7,9 \%$ & \multirow{5}{*}{$\begin{aligned} \mathrm{Chi}^{2} & =26.701 \\
p & =, 001\end{aligned}$} \\
\hline Prensa & $0,4 \%$ & $0,4 \%$ & $1,2 \%$ & \\
\hline Radio & $2,0 \%$ & $0,0 \%$ & $0,0 \%$ & \\
\hline Televisión & $7,5 \%$ & $0,0 \%$ & $0,0 \%$ & \\
\hline Otros & $9,4 \%$ & $0,0 \%$ & $0,4 \%$ & \\
\hline \multicolumn{5}{|c|}{ Los programas en la televisión tienen contenido sexual } \\
\hline Definitivamente sí & $15,7 \%$ & $5,1 \%$ & $6,3 \%$ & \multirow{5}{*}{$\begin{aligned} \mathrm{Chi}^{2} & =39,295 \\
p & =, 000\end{aligned}$} \\
\hline Probablemente sí & $20,1 \%$ & $2,0 \%$ & $2,0 \%$ & \\
\hline Indeciso & $24,0 \%$ & $0,4 \%$ & $0,8 \%$ & \\
\hline Probablemente no & $10,6 \%$ & $0,8 \%$ & $0,0 \%$ & \\
\hline Definitivamente no & $11,0 \%$ & $0,8 \%$ & $0,4 \%$ & \\
\hline \multicolumn{5}{|c|}{ Servicios de salud para adolescentes } \\
\hline No acude & $42,5 \%$ & $4,3 \%$ & $2,8 \%$ & \multirow{3}{*}{$\begin{aligned} \mathrm{Chi}^{2} & =10,069 \\
\mathrm{p} & =, 01\end{aligned}$} \\
\hline Algunas veces & $35,0 \%$ & $4,7 \%$ & $5,1 \%$ & \\
\hline Siempre & $3,9 \%$ & $0,0 \%$ & $1,6 \%$ & \\
\hline
\end{tabular}

17 años, exponiéndose a un riesgo reproductivo de un embarazo precoz y producir morbimortalidad materno perinatal ${ }^{(23,24)}$. Respecto al uso de métodos anticonceptivos, como el preservativo, píldora e inyectable, se observa que muchos de ellos no responden, relacionándose a un regular conocimiento en educación sexual ${ }^{(25)}$. En cuanto al reconocimiento de infecciones de transmisión sexual en la mujer, el resultado es de moderado a poco conocimiento y en varón es de poco a moderado conocimiento; observándose que la mayoría de los adolescentes no tienen la información sobre los signos y síntomas de la infección de transmisión sexual, lo cual nos alerta sí, como resultado, no buscarán atención oportuna. Álvarez et al. explican que el desconocimiento de las infecciones traerá complicaciones tardías, así como inflamaciones pélvicas crónicas, diseminación de verrugas genitales, cáncer cérvico uterino hasta infertilidad ${ }^{(16)}$. Este estudio aporta datos en cuanto al ofrecimiento de alcohol, encontrándose un resultado significativo, ya que se observa que, al menos una vez, le ofrecieron alcohol, Valbuena y Cueto hacen referencia al consumo de alcohol, relacionándolas a las conductas de riesgo, incrementándose las relaciones no planificadas y sin protección e iniciando su consumo en la etapa de la adolescencia ${ }^{(26-28)}$

En el ámbito familiar, aparecieron diferencias significativas según el grado de instrucción del padre y de la madre. Este último se observa de secundaria a primaria, en estudios realizados por Cueto, quien indica la existencia de una relación significativa de madres que no concluyen la secundaria completa ${ }^{(27)}$, por falta de 
Tabla 6. Resultados de regresión múltiple de los encuestados como variable dependiente de la actividad sexual

\begin{tabular}{|c|c|c|c|c|c|}
\hline \multirow{2}{*}{ Modelo } & \multicolumn{2}{|c|}{$\begin{array}{c}\text { Coeficientes } \\
\text { no estandarizados }\end{array}$} & \multirow{2}{*}{$\begin{array}{c}\begin{array}{c}\text { Coeficientes } \\
\text { estandarizados }\end{array} \\
\text { Beta }\end{array}$} & \multirow{2}{*}{$t$} & \multirow{2}{*}{ Valor-p } \\
\hline & B & Error estándar & & & \\
\hline Actividad sexual & 724 & 142 & Beta & 5,090 & 0,000 \\
\hline Apoyo de mamá & -123 & 038 & -200 & $-3,247$ & 0,001 \\
\hline Actividad sexual & 829 & 148 & & 5,620 & 0,000 \\
\hline Apoyo de mamá & -121 & 038 & -197 & $-3,223$ & 0,001 \\
\hline $\begin{array}{l}\text { Medio de comunicación que promueve más } \\
\text { las relaciones sexuales }\end{array}$ & -067 & 028 & -147 & $-2,408$ & 0,017 \\
\hline
\end{tabular}

apoyo de los padres; asimismo se observa que la madre tiene una actitud "regular", de "muy bien" a "bien", y el padre tiene una conducta "regular", "bien", "muy bien", y "muy mal". Estos aspectos influyen en el comportamiento sexual de los adolescentes, ya que la falta de un buen dialogo de padres a hijos genera carencias afectivas que el adolescente no sabe cómo resolver en una situación determinada. En cambio, sí se genera confianza con los padres, esto permitirá guiarlos en forma segura y efectiva, promoviendo practicas seguras y el uso de los métodos anticonceptivos; logrando de este modo su desarrollo potencial humano en la sociedad ${ }^{(29,30)}$. Otro aspecto de la conducta familiar es la exposición constante a la violencia física y verbal hacia el adolescente, lo cual tiene alcances negativos en la salud de ellos ${ }^{(31)}$. En estudios realizados se observa que los adolescentes que no reciben afecto y cuidado por parte de sus padres, prefieren salir de su domicilio $y$, posteriormente, se exponen a conductas de riesgo ${ }^{(32)}$.

En el contexto social, la comunicación con los amigos sobre temas de sexualidad y la curiosidad de experimentar las relaciones sexuales se asocian significativamente con el inicio de la actividad sexual Precoz. El estudiante está, por lo tanto, supeditado a sus pares respecto a su sexualidad y los valores aprendidos en la familia son importantes en la interacción con sus amigos ${ }^{(33)}$. Los medios de comunicación promueven las relaciones sexuales, tienen en ese sentido una fuerte significación, indicando la mayoría el internet y también los programas de televisión ${ }^{(34,35)}$.

Respecto al acceso a los servicios de salud al adolescente, los estudiantes no acuden a los mismos; otros manifiestan "algunas veces". En estudios realizados por Reyes et al. Los estudiantes comentan que las malas experiencias y el maltrato en la atención recibida son un condicionante negativo $y$, como resultado, no concurren a solicitar los servicios diferenciados para adolescentes ${ }^{(22)}$. La nueva normativa de salud para la atención integral del adolescente indica que "la oferta de métodos anticonceptivos deben estar disponibles en el servicio si el adolescente la/lo solicite y accedan a los servicios de salud sexual y salud reproductiva"(36).

La investigación se efectuó con procedimientos metodológicos rigurosos, la metodología cualitativa habría ayudado a fortalecer las conceptualizaciones y necesidades de la salud sexual reproductiva; siendo esto, sin duda, de interés en las políticas públicas en nuestro país.

Finalmente se concluye que los determinantes personales que condicionan la actividad sexual de los adolescentes constituyen un riesgo en su salud. El desempeño de la familia es el vínculo vital, así como el apoyo y el afecto que favorecen el desarrollo integral de la persona junto a la influencia de los amigos en la toma de decisiones y los medios de comunicación. A estos se suman la no asistencia a los servicios de salud que condicionan, todos ellos, el inicio de la actividad sexual de los adolescentes.

Se recomienda la identificación de estos condicionantes refuerza la necesidad de implementar estrategias para la promoción de la educación sexual integral, con la participación de la familia, la escuela y la comunidad, con enfoque de derechos, género e interculturalidad, con la participación de profesionales capacitados en salud sexual y reproductiva.

Agradecimientos: a los directores de los colegios que facilitaron el acceso para la presente Investigación y a los adolescentes que libremente participaron, por su generosa colaboración.

\section{REFERENCIAS BIBLIOGRÁFICAS}

1. Pineda S, Aliño M. El concepto de adolescencia. En: Márquez R. Manual de Prácticas Clínicas para la atención integral a la Salud en la Adolescencia. Cuba: MINSAP; 1999: 15-23. 
2. Fondo de las Naciones Unidas para la Infancia (UNICEF) Salud Adolescente [Internet] [Consultado 2019 Set 28] Disponible en: https://www.unicef.org/argentina/media/1546/file/Salud\%20adolescente.pdf

3. Royuela P, Rodriguez L, Marugan J, Carbajosa V. Factores de riesgo de la precocidad sexual en adolescentes. Rev Pediatr Aten Primaria. 2015; 17(66): 127-36.

4. Paulin-Holguín Y, Mendoza L, Esquivel C, R. Sánchez-M, Daraviña AF, Acuña M. Factores asociados al inicio de la actividad sexual en adolescentes de Tuluá, Colombia. Revi Chil Obs Ginecol. 2013; 78(3): 209-19.

5. Electra GA, Temístocles MG, Adela MV, Vania MN. Factores familiares asociados al inicio sexual temprano en adolescentes consultantes en un centro de salud sexual y reproductiva en Santiago de Chile. Rev Med Chil. 2013; 141(3): 313-9.

6. Mendoza LA, Claros DI, Peñaranda CB. Actividad sexual temprana y embarazo en la adolescencia: estado del arte. Rev Chil Obs Ginecol. 2016; 81(3): 243-53.

7. Rengifo S, Uribe V, Yporra K. Inicio temprano de relaciones sexuales en adolescentes escolares de la ciudad de Ica, 2014. Rev méd panacea. 2014; 4(1): 8-12.

8. Carmona G, Beltran J, Calderón M, Piazza M, Chávez S. Contextualization of sexual initiation and individual barriers to the use of contraceptives by adolescents of Lima, Huamanga, and lquitos. Rev. Perú Med Exp Salud Pública. 2017; 34(4): 601-10.

9. Apoyo a programas de población (APROPO). La sexualidad [Internet] [Consultado 2019 Jul 15] Disponible en: https://www.apropo.org.pe/wp-content/uploads/2017/08/ MEMORIA-FINAL-2013-PS.pdf

10. Calero E, Sandra R, Aniocha T. Abordaje de la sexualidad en la adolescencia. Rev Humanidades Médicas. 2017; 2(2): 21-4.

11. Campo-Arias A, Silva J, Mariluz M, Melany C, Paola N. Factores asociados con el inicio temprano de relaciones sexuales en estudiantes adolescentes de un colegio de Bucaramanga, Colombia. Rev Colomb Psiquiatr. 2004; 33(4): 367-377.

12. Apaza LC. Factores individuales y familiares para el inicio de la actividad sexual coital en estudiantes de la Institución Educativa $N^{\circ} 1178$ Javier Heraud de San Juan De Lurigancho, octubre 2016 [Internet] Lima: Universidad Nacional Mayor de San Marcos; 2017 [Consultado 2019 Dic 3] Disponible en: https://core.ac.uk/download/ pdf/323353363.pdf

13. Ordoñez J, Real J, Gallardo J, Alvarado H, Roby A. Conocimientos sobre salud sexual y su relación el comportamiento sexual en estudiantes universitarios. Univ Nac Guayaquil, Ecuador. 2017; 78(4): 419-23.

14. Montero A. Anticoncepción en la adolescencia. Rev. Médica Clínica Las Condes. 2011; 22(1): 59-67.

15. Villalobos A, de Castro F, Rojas R, Allen B. Anticoncepción en adolescentes mexicanos de escuelas del nivel medio superior: Uso y necesidades insatisfechas. Salud Pública Mex. 2017; 59(5): 566-76.

16. Álvarez M, Gómez JD, De La Torre Navarro LM. Factores relacionados con el contagio de las infecciones de transmisión sexual en la adolescencia. Rev. Habanera Ciencias Médicas. 2014; 13(2): 270-83.

17. Ortiz- Melgar M, Pérez-Saavedra V, Valentín-Ballarta JJ, Zúñiga- Hurtado AL. Asociación entre consumo de alcohol y relaciones sexuales ocasionales en los adolescentes. Rev. Enferm Hered. 2016; 8(2): 109-114.

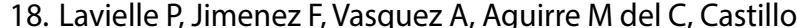
$M$, Vega S. Impacto de la familia en las conductas sexuales de riesgo de los adolescentes. Rev Medica del Inst Mex del Seguro Soc. 2014; 52: 38-43.

19. Cabrera V, Docal MC, Manrique LM, Cortes J, Tobón C. Familia y escuela: contextos asociados al inicio de la actividad sexual de los adolescentes colombianos. Rev Salud Pública. 2018; 20(3): 279-85.

20. Leal I, Molina FT, Luttges GC, González DE, Gonzalez A. Edad de inicio sexual y asociación a variables de salud sexual y violencia en la relación de pareja en adolescentes chilenos. Rev. Chil Obs Ginecol. 2018; 83(2): 149-60.

21. Figueroa L, Figueroa L. Conductas sexuales de riesgo en adolescentes desde el contexto cubano. Rev. Ciencias Médicas Pinar del Rio. 2017; 21(2): 193-301.

22. De Jesús-Reyes D, Menkes-Bancet $C$, Meza-Palmeros JA. Acceso y atención en servicios de salud en adolescentes de escuelas públicas de Nuevo León, México. Univ Costa Rica. 2016; 13(2): 1-23.

23. López LL. Factores Sociales que se sitúan en un contexto de vulnerabilidad a adolescentes sobrevivientes de violencia sexual del Alberge Lazos de Amor, zona 1 Ciudad Capital [Internet] Guatemala de La Asunción: Universidad Rafael Landívar; 2014 [Consultado 2019 Dic 10] Disponible en: http://biblio3.url.edu.gt/Tesario/2014/05/67/Lopez-Ligia.pdf

24. Soriano-Ayala E, González-Jiménez AJ, Soriano-Ferrer M. Educación para la salud sexual del enamoramiento al aborto. Un estudio cualitativo con adolescentes españoles e inmigrantes. Perfiles Educativos. 2014; 36(144): 105-119

25. Fernández-Honorio I. Conocimiento y uso de los métodos anticonceptivos en adolescentes de centros educativos del distrito de San Juan de Lurigancho. Rev. Cient Agora. 2015; 02(01): 79-88.

26. Lestón R. Educacion Sexual en la Adolescencia: propuesta de intervención desde la Educación Social. 2015 [Internet] Palencia: Universidad de Valladolid; 2015 [Consultado 2019 Dic 12] Disponible en: http://uvadoc.uva. es/handle/10324/15985

27. Cueto S, Leon J. Early sexual initiation among adolescents: A longitudinal analysis for 15-year-olds in Peru. Interam J Psychol. 2016; 50(2): 186-203.

28. Morales J, Tuse-Medina R, Carcausto W. Consumo de Alcohol y drogas Ilicitas en adolescentes preuniversitarios. Rev Cuba Med Gen Integr. 2019; 35(3): 1-16.

29. Domínguez I. Influencia de la familia en la sexualidad adolescente. Rev Cuba Obstet y Ginecol. 2011; 37(3): 387-398.

30. Rivera-Rivera L, Leyva-López A, García-Guerra A, de Castro F, González-Hernández D, de los Santos LM. Inicio de relaciones sexuales con penetración y factores asociados en chicos y chicas de México de 14-19 años de edad con escolarización en centros públicos. Gac Sanit. 2016 Ene $1 ;$ 30(1): 24-30.

31. Flórez CE. Factores socioeconómicos y contextuales que determinan la actividad reproductiva de las adolescentes en Colombia. Rev. Panam Salud Publica/Pan Am J Public Heal. 2005; 18(6): 388-402.

32. Pinzón ÁM, Ruiz ÁM, Aguilera- PA, Abril PD. Factores asociados al inicio de vida sexual y al embarazo adolescente en Colombia. Estudio de corte transversal. Rev. Chil Obs Ginecol. 2018; 83(5): 487-499. 
33. Rivera D, Proaño E. La Influencia de los Pares y la Familia en el Inicio de la Actividad Sexual en Adolescentes. Augusto Guzzo Rev. Académica. 2017; 1(19): 39-53.

34. Valdez C, Benavides R, González V, Onofre D, Castillo $L$, et al. Internet y conducta sexual de riesgo para VIH/SIDA en jóvenes. Rev. Electron Trimest Enfermaeri [Internet] 2015; 38: 160-8[Consultado 2020 Jun 20] Disponible en: http://scielo.isciii.es/pdf/eg/v14n38/ en_docencia3.pdf
35. Ruiz-Canela M, López-Del Burgo C, Carlos S, Calatrava M, Osorio A, De Irala J. Familia, amigos y otras fuentes de información asociadas al inicio de las relaciones sexuales en adolescentes de El Salvador. Rev. Panam Salud Publica/Pan Am J Public Heal. 2012; 31(1): 54-61.

36. Ministerio de Salud. Norma Técnica De Salud Para La Atención Integral De Salud De Adolescentes [Internet] [Consultado 2020 Jul 03] Disponible en: http://bvs.minsa.gob.pe/local/MINSA/4942.pdf 